\title{
The effectiveness of home-based nursing intervention in the elderly with recurrent diabetic foot ulcers: A case report
}

\author{
Annisa Wuri Kartika, ${ }^{1}$ Widyatuti Widyatuti, ${ }^{2}$ Etty Rekawati ${ }^{2}$ \\ ${ }^{1}$ School of Nursing, Faculty of Medicine, Universitas Brawijaya, Malang; ${ }^{2}$ Community Health Nursing \\ Department, Faculty of Nursing, Universitas Indonesia, Depok, Indonesia
}

\begin{abstract}
Background: Inadequate glycemic control usually leads to peripheral neuropathy, vasculopathy, and foot deformities that leads to diabetic foot ulcer (DFU), and a 10-years history of diabetes and inadequate self-management increases the risk of reoccurring DFU. A home-based intervention program, which includes Diabetes Self-Management Education (DSME) and wound care is the most likely approach, to engage families and overcoming the barriers in self-care management. The aim of this study is to provide an overview on the effectiveness of home-based nursing intervention in the elderly patients with reoccurring diabetic foot ulcers.
\end{abstract}

Design and method: In this study, a case study approach was used that presented a case related to self-management and wound care in elderly patients with reoccurring DFU. The intervention was carried out by the community health nurses for eight weeks with home visit.

Results: Significant changes were reported in diabetes selfmanagement practices, blood glucose level, and wound healing. The Diabetes Self-Management Questionnaire (DSMQ) score increased from 5.62 to 8.,54 and the Summary of Diabetes SelfCare Activities (SDSCA) score increased from 3.2 to 6 . The Bates Jansen Examination score decreased from 24 and 26 to 17 and 14 in six weeks.

Conclusions: The results confirm that family-based interventions program are effective in improving glycemic control and wound healing. Community nursing is believed to prevent an increase in self-management behavior to prevent recurring DFU and maintain a healthy life-style about diabetes self-management education.

\section{Introduction}

Diabetes Mellitus (DM) is a metabolic disease with a large increase in prevalence, especially in a developing country. ${ }^{1}$ In Indonesia, basic health research reported that the number of DM increased from $6.9 \%$ in 2013 to $8.5 \%$ in $2018 .^{2}$ Self-management is an integral part of diabetes management procedures, $98 \%$ of which are self-care procedures that include supporting self-care activities, including self-monitoring of blood glucose, low-fat diet, daily exercise, and implementing strategic to prevent foot ulcers. Research has shown that many of these self-care activities are independent of one another. ${ }^{3,4}$ Diabetes management practice is not only complex but also demanding, therefore majority of the patients find it difficult to modify lifestyle and to achieve important physical and psychological impact. ${ }^{5}$ With the highest prevalence in incidence, older patients with diabetes encountered many difficulties in self-care activity due to aging process, such as cognitive dysfunction, functional impairment, polypharmacy, vision, and hearing impairment, and depression. ${ }^{6}$ In addition, caregivers or families of older people should be included in the intervention program in order to improve self-management behavior. A home based intervention programs for self-management education with Diabetes Self-Management Education (DSME) showed significant effect in glycemic control, self-efficacy, and psychosocial improvements. ${ }^{7-9}$

The rise in blood glucose level in the elderly usually leads to complications of various pathophysiological systems, such as peripheral neuropathy, vasculopathy, and foot deformities that lead to a diabetic foot ulcer (DFU). ${ }^{9,10}$ DFU are classified as chronic, non-healing wounds that create a disruption or an injury to skin layers, necrosis or gangrene that usually occur on the soles of the feet, as a result of peripheral neuropathy or peripheral arterial disease. Sensory neuropathy is the main and most important causative factor, which is usually deep $(>50 \%)$ before experiencing a loss of protective sensation resulting in susceptibility to physical and thermal trauma and increase the risk of foot ulcers. ${ }^{11}$ DFU risk factors include: male sex, diabetes more than ten years, peripheral neuropathy, abnormal foot structure (bone alterations, calluses, nails thickening), peripheral arterial disease, smoking, history of ulcers or amputation, and inadequate glycemic control. ${ }^{12}$ As the incidence of diabetes increases, it also predict an increase in complications in diabetes such as DFU, which affects approximately $15-25 \%$ of diabetics during lifetime. ${ }^{13,12}$ With a

Significance for public health

Diabetes mellitus (DM) with diabetic foot ulcer significantly contributes to the deterioration in health and financial burden on the patient. The increased risk of diabetic foot ulcers, especially in elderly patients with inadequate glycemic control tends to increase diabetes complications and mortality. Furthermore, the functional impairment, cognitive dysfunction, and poverty caused by the aging process make it difficult for elderly people to access health services. Home-based interventions carried out by community health nurses using the patient-centered care approach have advantages in improving access to services. Focusing on the self-management with Diabetes Self-Management Education (DSME) and wound care management to improve glycemic control, optimize the healing process, and also improve health promotion to prevent recurrent diabetic foot ulcers. This article provides an overview on the effectiveness of home-based nursing intervention in the elderly patients with recurrent diabetic foot ulcers. 
global prevalence of $6.3 \%$, DFU is the precursor to approximately $85 \%$ of the lower extremity amputation. ${ }^{14}$

Diabetic patients with DFU also associated with peripheral arterial disease (PAD), increased mortality, reduced mobility, poor psychosocial adjustment, and reoccurrence of ulcer. ${ }^{15,16}$ caused by biological, physical or behavioral factors, such as increased plant stress, loss the "gift of pain" which leads to lack of appropriate preventive measures, and no follow-up care to identify warning signs of recurrence. Treatment of reoccurring diabetic foot ulcer required adequate glycemic control, ongoing professional foot care at intervals of 1 to 3 months and properly fitting footwear. ${ }^{16}$ The ability to identify patients at high risk of this complication can help healthcare providers focus on preventive interventions. The patient education program in treatment of hyperglycemia and integrated foot care is also an important factor to prevent foot ulcer pain. Treatment of hyperglycemia and appropriate wound care management will decrease the complications of DFU. The aim of this study is to provide an overview on the effectiveness of home-based nursing intervention in the elderly patients with reoccurring diabetic foot ulcers.

\section{Design and Methods}

This is a case report, and the participants were selected by purposive sampling and given informed consent to attend this study, which was conducted from November to December 2019. The participant is a 69-year-old male patient diagnosed with diabetes mellitus type 2 in 2005 (15 years). He was on medication, using metformin $500 \mathrm{mg}$ twice daily and the last visit to the physician was 5 months ago, and considering visitation for medical services just in case there is a symptom and buying medicine from a pharmacy (refers to an old recipe). There is no dietary abstinence, but the patient said he avoid sugary drinks and limit the consumption of rice. The daily activities are gardening and teaching Qor'an. For the past three months, the average blood glucose level was 198 $\mathrm{mg} / \mathrm{dl}$. At the first meeting in home visit, blood glucose in postprandial level $=315 \mathrm{mg} / \mathrm{dl}$ and cholesterol level $=198 \mathrm{mg} / \mathrm{dl}$. This high value of blood glucose is influenced by the patient inappropriate habits in taking medication and eating behavior. He said that he did not usually eat breakfast, only consumed cassava or other traditional snacks, which is generally made from wheat or rice flour. He only eats rice during the day and night, in such a way that the medication is taken during the day and night. And often the drugs that must be taken during the day are not taken because they are forgotten. Improper medication and inadequate eating behavior coupled with a lack of physical activity is the main factor for inad- equate glycemic control.

The patient has experienced a diabetic foot ulcer twice in the last three years and suffered amputation in-ring toe two years ago. Due to his present condition, he has experienced a diabetic wound in his left foot since last month. From Figure 1, it can be concluded that the location of his wound is in his sole and pinkie toe. The wound examinations are grade 2 in his pinkie toe, while the wound in his sole-foot is in grade 1 (Wagner classification). There was callus but the stink is not reported, edema in the left foot, dry skin, and the integument color is dark (not like the other's skin). Bates Jensen Examination of the wound in sole foot with total score $=24$ and wound in pinkie toe with total score $=26$. There was edema in the left foot. From observation and interview, it was discovered that the patient has a habit of sitting with one of the legs (especially leg with diabetic foot ulcer) raised in the chair or crossed the legs. The activity in teaching Qor'an was carried out by sitting in a cross-legged position with a duration of 2-3 $\mathrm{h}$ daily. This position affects blood circulation, which reduces blood flow to the legs area, especially the area with diabetic foot ulcers, and can hinder the healing process.

Nursing care is provided by scheduling a visit to homes twice a week for 6 weeks. Intervention was carried out by DSME and also wound care. Patient and family member as caregiver consisting of wife and daughter involved in health education. DSME delivered information about self-management, which includes understanding diabetes, healthy eating, physical activities, understanding and monitoring blood glucose, medication adherence, problem-solving, coping, and foot care. The education program used interactive discussions and exchange of experiences related to self-management. Education about medication is delivered by teaching and supporting the patient with pill container and record the activity in the medication logbook, which was evaluated by a nurse on a weekly basis. In terms of diet management, the carbohydrate counting method is used to assess the patient carbohydrate requirement and measure the portion of meals daily. This result is complemented by creating a meal schedule with a menu tailored to patient eating habits and recorded in the logbook. The physical activity recommendation was carried out in the fourth week after reduced edema by doing light physical activity by stretching and brisk walking every morning after praying (Shubuh) for $30 \mathrm{~min}$ daily. The ability of self-care was measured by the Diabetes Selfmanagement Questionnaire (DSMQ), blood glucose level, and The Summary of Diabetes Self-Care Activities Measure (SDSCA) before and after the intervention.

Wound care management consists of wound care practice with modern dressing. A low adherence dressing helps in distributing the fluid, and for the edema in the left foot, a bandage was used for

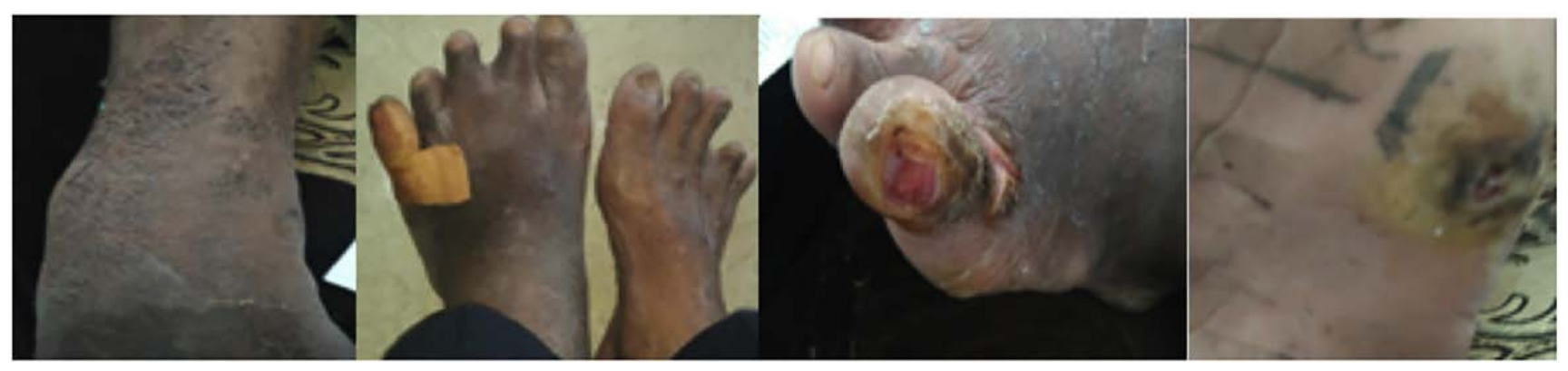

Figure 1. First inspection of wound (5 November 2019). 
compression. In the fifth week, alternative wound care materials are used, such as honey as a bandage and baby soap as a wound washer, to reduce costs due to the patient's economic situation. The patient was also encouraged to perform regular foot care, which includes washing of foot regularly, changing the sitting habit, and wearing fitting footwear during daily activities. The management of DFU was measured by the total score of Bates Jansen Examination before and after the intervention.

\section{Results and Discussions}

Table 1 lists the DSMQ and SDSCA scores, which describe the knowledge, attitudes, and practice of self-management. The DSMQ score in the first assessment describes that the patient ability to carry out self-care activities is quite good. The highest DSMQ score is for diet control and health care on the subscale and the lowest is for physical activity and glucose management on the subscale. The patient's nearly 15-year history in DM provides an opportunity to understand the disease and experience in terms of

Table 1. DSMQ and SDSCA pre and post DSME. Parameters Pre Post

Diabetes Self-management Questionnaire (DSMQ) 5.628 .54 The Summary of Diabetes Self-Care Activities Measure (SDSCA) $3.2 \quad 6$

Post pandrial blood glucose

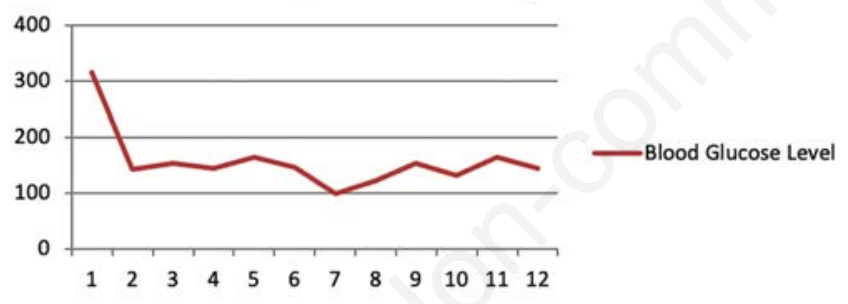

Figure 2. Blood glucose level. autonomous management. The patient is experienced in identifying hyperglycemic conditions by noticing the symptoms, namely frequent urination and drowsiness in such a way that they respond to taking medication. However, this was not followed by other self-maintenance behaviors, such as diet and physical activity because they felt that taking medication alone was enough to overcome the disease. Health education that is delivered with discussion strategies and sharing experiences has a good impact on the implementation of patient-centered care strategies. This strategy provides the opportunity for nurses to provide interventions in accordance with the patient conditions. There was a significant increase in the DSMQ score after training at week 6 with a score of 8.54 and a better score on all four subscales. In terms of practice with SDSCA, the scoring before intervention was low with the lowest indicator as physical activity, foot care, and diet. The results after intervention increased with more regular self-management practices after being controlled by the nurse and caregiver. Better self-management practices include taking medications on a timely basis, maintaining regular foot care, and healthy eating habits. In terms of physical activity, the program is limited to walking due to a diabetic foot ulcer.

Figure 2 contains a description of the blood sugar level, which shows the significant decrease in value and stability in 12 measurements (twice a week for 6 weeks). This suggests that blood sugar control has improved along with better self-management practices, especially in regard to taking medication and diet management. The development in 2 weeks of wound care management in Figure 3 showed that compression reduces the edema. The patients were thought how to apply virgin coconut oil (VCO) to optimize moisturizer in dry skin. During 4 weeks of wound care practice in figure 4 , the result showed changes that were consistent with wound examination, that is a total score of Bates Jansen Examination of the wound in sole foot is $=14$. And the wound in the pinkie toe has total score $=17$. For the following weeks, baby soap was recommended and honey as a substitution for washing and dressing. This product is easy to obtain, cheap, and easy to use. The wound has changed in size, depth, cave size, the decreasing in necrosis tissue, skin color around the wound by decreasing callus. However, there was little development for the blood circulation (edema), and the problem was bad habits in patient behavior, which includes nonmaintenance of the bandage (removed when bathing), that does not change the activity especially in sitting position, or not maintaining the elevation of the foot. It is important to inform the patient on the use bandage, and other bad habits that must be changed by the patient sitting habits and activities that cause blood flow obstruc-

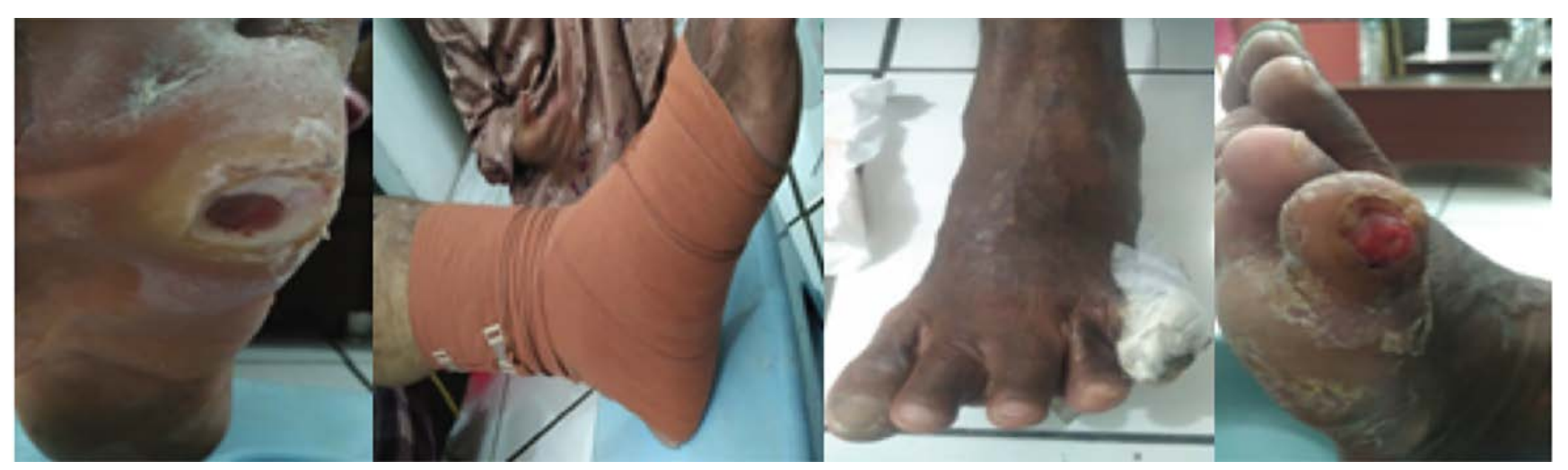

Figure 3. Wound on 16 November 2019. 
tion, for example, posture while sitting, standing, and keeping the legs higher with the pillows while sleeping. The adherence in nursing advice increased in $3^{\text {rd }}$ week after regular supervision from nurse and caregiver. It was discovered that the compliance of patients and family to keep maintaining bandage is the important factor to reduce edema.

Health education is an effective strategy to improve self-care skills including self-management behavior, HbAlc, and blood sugar control, body weight, improve quality of life, decrease stress and increase adherence to medication behavior., ${ }^{4,5,17-19}$ Therefore, health education with DSME provides an advantage for patients in order to optimize the 3 components of behavior in self-care of chronic illness. According to the research conducted by Abdulrahman et al. ${ }^{20}$ provides results that increasing knowledge with health education will increase understanding on the importance of self-care followed by increased behavior. Although health education has a great impact on patient perception, in some condition there is poor understanding in patient especially those with low education and cognitive impairment, such as elderly. When the information is not well communicated by the healthcare professional, patients can have different perceptions and draw their own conclusions. ${ }^{21}$ In this case, the patient has a misunderstanding of the correct schedule because, according to the patient, nutrition is only included in rice consumption, although he has a snack in the morning that contain carbohydrate but did not think that he must be taking medication. The health education must consider patient perception in such a way that the nurses can give better strategies to delivered information with proper communication techniques. After an intervention with DSME, changes in self-maintenance behavior can be improved by direct supervision on weekly basis uses a logbook. Home-based intervention program gives an advantage for nurses because they are more informed about habits, direct observation of behavior, more time for education, and involving families in the intervention. ${ }^{7,22}$ The results support the value of family-based interventions to improve diabetes selfmanagement. ${ }^{22}$ The important thing is to integrate DSME in home visit program according to the national program and to adapt the teaching material to the socio-economic context, and to culture and literacy level of the target groups. ${ }^{23}$

Wound care is a large part of the growing workload of community nurses, especially with DM-related wounds. There is a need to find a simple and inexpensive product that can be administered to patients for self-care, therefore reducing the workload of the nurse's and encouraging patients to participate in the wound healing process. For product availability and ease of use, regular salt and soap are safe, simple, and do not cause harm or allergies when compared to povidone iodine and normal saline. ${ }^{23}$ This method does not need long-term hospitalization and reduces expenses. Soap is an antiseptic substance and there is no soap-related harm reported till now, and no complications have been reported with water and soap. ${ }^{23}$ The other product used for wound care is honey, and Medicinal honey products are an excellent resource for nurses in the community because of the bandages' multifactorial effects, which often leads to being referred to as all-in-one prep bandages and effective wound bed preparation. These include antimicrobial action, deodorization, pH-modulating agent, debridement, antiinflammatory action, scar reduction, stimulation of new tissue growth (angiogenesis), biofilm control effect, pain management. ${ }^{24}$

Early detection and case finding is an important factor in reducing the risk of diabetic foot recurrence. Assessment of knowledge, history of ulcer, and behavior are important factors in determining effective interventions. Patients' beliefs regarding the symptoms of ulceration and their perceived personal control over ulceration are important determinants of foot care. ${ }^{25}$ Increasing knowledge and life-style modification practice include healthy eating, foot care, and properly fitting footwear are predicted to prevent foot ulcer recurrence. ${ }^{16}$ Adequate glycemic control, regular foot care and early detection of the symptom recurrence such as a new lesion, callus, and foot deformities are key component included in DSME.

\section{Conclusions}

Home-based interventions play an important role in improving patient and family self-care management with mobility impairment due to DFU and the elderly. Strengthening families through involvement in each intervention can help assist patient with monitoring. Understanding the reasons for the recurrence of diabetic ulcers by early assessment of biological and behavioral factors is important to prevent the remission. Increasing foot care practices and behavior changes are the key component to reduce the risk of ulcer recurrence and reduce the burden on patient and families.
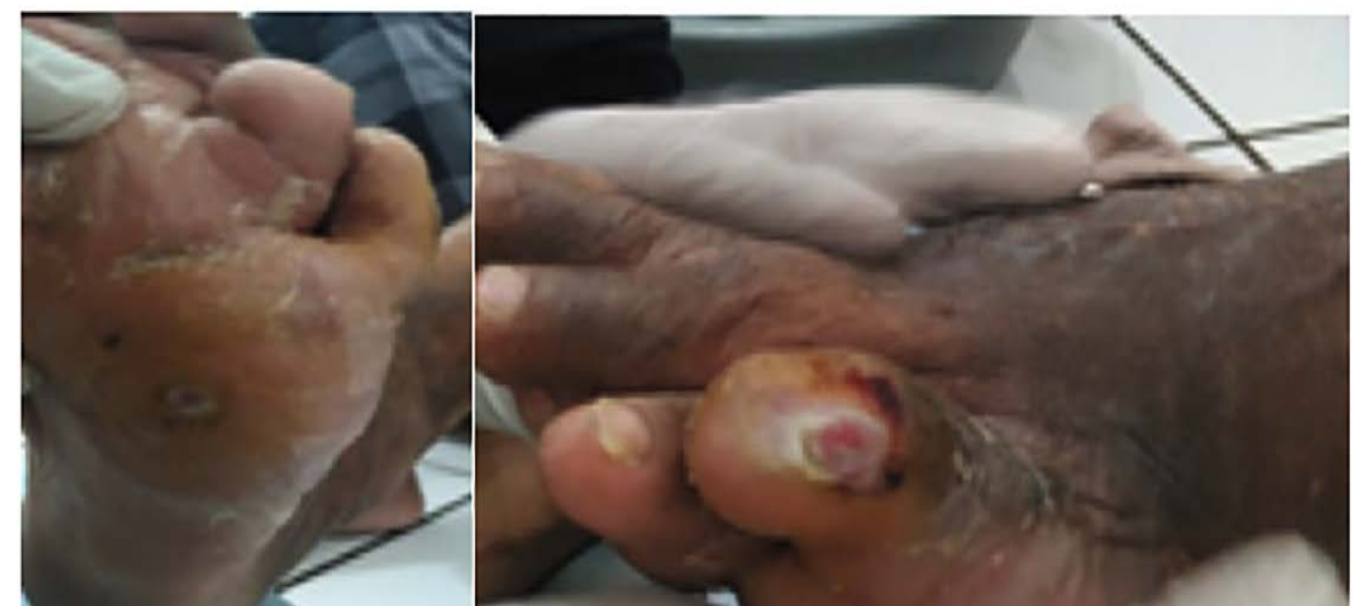

Figure 4. Wound on 30 November 2019. 
Correspondence: Annisa Wuri Kartika, School of Nursing, Faculty of Medicine, Universitas Brawijaya Malang, Jl Puncak Dieng, Kalisongo, Kecamatan Dau Malang, East Java 65151, Indonesia. Tel. +62.341569117 - Fax: +62.341564755

E-mail: annisa_tika@ub.ac.id

Key words: Home-based intervention; self-care management; foot ulcer recurrence; DSME.

Contributions: All authors contributed equally to this article. AWK conducted this study, WW and ER served as supervisors and reviewed the article.

Conflict of interest: The authors declare that there was no potential conflict of interest.

Funding: This study was financially supported by Faculty of Medicine Brawijaya University Malang.

Acknowledgement: The authors express profound gratitude to all those that contributed to the completion of the study. Many thanks to the patient for the willingness to participate. Thanks to Sukmajaya Primary Health Care, Faculty of Nursing Universitas Indonesia and School of Nursing, Faculty of Medicine, Universitas Brawijaya for the support during this study.

Conference presentation: Part of this study was presented at the $1^{\text {st }}$ International Nursing and Health Sciences Symposium, November $13^{\text {th }}$ to $15^{\text {th }} 2020$, Brawijaya University, Malang, Indonesia.

Received for publication: 14 January 2021.

Accepted for publication: 18 March 2021.

oCopyright: the Author(s), 2021

Licensee PAGEPress, Italy

Journal of Public Health Research 2021;10:2162

doi:10.4081/jphr.2021.2162

This work is licensed under a Creative Commons Attribution NonCommercial 4.0 License (CC BY-NC 4.0).

\section{References}

1. Wu Y, Ding Y, Tanaka Y, et al. Risk factors contributing to type 2 diabetes and recent advances in the treatment and prevention. Int J Med Sci 2014;11:1185-200.

2. Ministry of Health Republic of Indonesia. Basic Health Research 2018. Jakarta: Ministry of Health Republic of Indonesia; 2018.

3. Jannoo Z, Mamode Khan N. Medication adherence and diabetes self-care activities among patients with type 2 diabetes mellitus. Value Heal Reg Issues 2019;18:30-5.

4. Yuan C, Lai CW, Chan LW, et al. The effect of diabetes selfmanagement education on body weight, glycemic control, and other metabolic markers in patients with type 2 diabetes mellitus. J Diabetes Res 2014;2014:789761.

5. Trevisan DD, São-João T, Cornélio $M$, et al. Effect of an 'implementation intention' intervention on adherence to oral anti-diabetic medication in Brazilians with type 2 diabetes. Patient Educ Couns 2020;103:582-8.

6. Kirkman MS, Briscoe VJ, Clark N, et al. Diabetes in older adults. Diabetes Care 2012;35:2650-64.

7. García AA, Brown SA, Horner SD, et al. Home-based diabetes symptom self-management education for Mexican Americans with type 2 diabetes. Health Educ Res 2014;30:484-96.

8. Davis WA, Lewin G, Davis TME, et al. Determinants and costs of community nursing in patients with type 2 diabetes from a community-based observational study: The Fremantle Diabetes Study. Int J Nurs Stud 2013;50:1166-71.

9. Felix HC, Narcisse MR, Long CR, et al. The effect of family diabetes self-management education on self-care behaviors of Marshallese adults with type 2 diabetes. Am J Health Behav 2019;43:490-7.

10. Handayani E. Modern wound care application in diabetic wound management. Int J Res Med Sci 2017;5:702-6.

11. Gnanasundaram S, Ramalingam P, Nath B. Gait changes in persons with diabetes: early risk marker for diabetic foot ulcer. Foot Ankle Surg 2020;26:163-8.

12. Rosyid FN. Etiology, pathophysiology, diagnosis and management of diabetics' foot ulcer. Int J Res Med Sci 2017;5:420613.

13. Tresierra-Ayala MÁ, García Rojas A. Association between peripheral arterial disease and diabetic foot ulcers in patients with diabetes mellitus type 2. Medicina Universitaria 2017;19:123-6.

14. Yazdanpanah L, Shahbazian H, Nazari I, et al. Diabetes \& metabolic syndrome: Clinical research \& reviews risk factors associated with diabetic foot ulcer-free survival in patients with diabetes. Diabetes Metab Syndr Clin Res Rev 2018;12:1039-43.

15. Gianino E, Miller C, Gilmore J. Smart wound dressings for diabetic chronic wounds. Bioengineering (Basel) 2018;5:51.

16. Armstrong DG, Boulton AJM, Bus SA. Diabetic foot ulcers and their recurrence. N Engl J Med 2017;376:2367-75.

17. Yu CH, Parsons JA, Mamdani M, et al. A web-based intervention to support self-management of patients with type 2 diabetes mellitus: Effect on self-efficacy, self-care and diabetes distress. BMC Med Inform Decis Mak 2014;14:117.

18. Nwankwo CU, Ezenwaka CE, Onuoha PC, et al. Implementing diabetes self-management education (DSME) in a Nigerian population: Perceptions of practice nurses and dieticians. Arch Physiol Biochem 2015;121:123-7.

19. Lavelle D, Zeitoun J, Stern M, et al. Diabetes self management education in the home. Atl Heal Syst 2016;8:1-8.

20. Abdulrahman M, Husain ZSM, Abdouli KA, et al. Association between knowledge, awareness, and practice of patients with type 2 diabetes with socio-economic status, adherence to medication and disease complications. Diabetes Res Clin Pract 2020;163:108124.

21. Lukman NA, Leibing A, Merry L. Self-care experiences of adults with chronic disease in Indonesia: An integrative review. Int J Chronic Dis 2020;2020:1-17.

22. Dube L, Van den Broucke S, Dhoore W, et al. An audit of diabetes self-management education programs in South Africa. J Public Health Res 2015;4:581.

23. Najafian A, Fallahi S, Khorgoei T, et al. Role of soap and water in the treatment of wound dehiscence compared to normal saline plus povidone-iodine: A randomized clinical trial. J Educ Health Promot 2015;4:86.

24. Evans J. A solution to cost-effective wound management in the community. J Comm Nurs 2014;28:46-51.

25. Vedhara K, Dawe K, Wetherell MA, et al. Illness beliefs predict self-care behaviours in patients with diabetic foot ulcers: a prospective study. Diabetes Res Clin Pract 2014;106:67-72. 